\title{
¿CAMBIO CON TRANSFORMACIÓN? POLÍTICAS PÚBLICAS DE DESASTRE EN CHILE POST TERREMOTO 2010, UN CASO DE ESTUDIO
}

Cristóbal Mena A. ${ }^{1 *}$

\section{RESUMEN}

Este artículo examina el desarrollo de una ventana de oportunidad post-desastre para introducir un cambio en las Políticas Públicas de Desastre (PPD) tomando a Chile como caso de estudio. La investigación revela cómo el cambio de política pública se comporta luego del Terremoto del 27 de febrero de 2010 y desafía la suposición que el cambio, por sí mismo, mejora las condiciones previas de las condiciones no-estructurales como las políticas públicas. La investigación concluye que se ha producido un cambio sin transformación en el contexto chileno de PPD, debido a la carencia de emprendedores de política pública que permitan acoplar soluciones que impulsen una metamorfosis en la manera de gestionar el riesgo de desastres.

\section{PALABRAS CLAVE}

Políticas Públicas de Desastre, Reconstrucción, Cambio con Transformación, Gestión del Riesgo de Desastre, Cambio de Políticas Públicas

CHANGE WITH TRANSFORMATION? DISASTER PUBLIC POLICIES IN CHILE AFTER THE 2010 EARTHQUAKE, A CASE STUDY

\section{ABSTRACT}

This paper examines the development of a post-disaster window of opportunity to introduce change in Disaster Public Policy (DPP) taking Chile as a case study. The research reveals how policy change behaves after the 27th February 2010 Earthquake and challenges the assumption that change, by itself, improves the prior conditions of non-structural measures such as public policies. The research concludes that in the Chilean DPP context there is change without transformation due to the lack of disaster-policy entrepreneurs, who can couple solutions that produce metamorphosis in to reduce disaster risk.

\section{KEYWORDS}

Disaster Public Policies, Reconstruction, Change with Transformation, Disaster Risk Management, Policy Change
1. Consultor Independiente en Gestión del Riesgo de Desastre y Continuidad de Gobierno, Chile.

*Autor de correspondencia: cristobal.menaa@gmail.com

\section{RECIBIDO}

1o de octobre de 2017

\section{ACEPTADO}

22 de octobre de 2017

\section{PUBLICADO}

30 de enero de 2018

\section{Formato cita}

Recomendada (APA):

Mena A., C. (2018). ¿Cambio con transformación? Políticas Públicas de Desastre en Chile post Terremoto 2010, un caso de estudio. REDER, 2(1), pp.4-20.

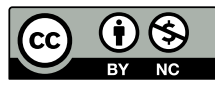

Todos los artículos publicados en REDER siguen una política de Acceso Abierto y se respaldan en una Licencia CreativeCommons Atribución-NoComercial 4.0 Internacional.

Revista de Estudios Latinoamericanos sobre Reducción del Riesgo de Desastres (REDER)

Diseño: Lupe Bezzina Tipografías: Hospital 


\section{INTRODUCCION}

Recuperarse de un desastre es habitualmente comprendido como la reconstrucción material de lo construido. Sin embargo, superar una catástrofe ${ }^{1}$ debe ser abordado no sólo como la reparación y mejora de las dimensiones estructurales (Tierney \& Oliver-Smith, 2012), sino también de las condiciones inmateriales que configuran la vulnerabilidad en un contexto social y geográfico determinado, fomentando la generación de un "ambiente de indagación" Por esto, la condiciones inmateriales deben ser interpretadas y proyectadas en diferentes dimensiones (Tironi, 2014) para hacer visibles los aspectos más invisibles que configuran la vulnerabilidad; tales como la estructura institucional y los valores políticos. En definitiva, la recuperación debe también considerar la revisión de las medidas no-estructurales (Platt, 1999) las políticas públicas de desastre (PPD), un término que hemos acuñado en esta investigación para describir aquellas políticas públicas (Kilpatrick, 2000) aplicadas a las fases del ciclo de la gestión del riesgo de desastre (GRD) (Alexander, 2002).

Ahora bien, los desastres pueden abrir una ventana de oportunidad (Birkmann et al., 2010; Davis \& Alexander, 2016; Pelling \& Dill, 2006; Wu \& Lindell, 2004) para observar la progresión interna de la vulnerabilidad (Wisner, Blaikie, Cannon, \& Davis, 2004) en una comunidad afectada por una catástrofe permitiendo a los emprendedores de política (Kingdon, 1995) introducir cambio en las PPD cuando estas no cumplen las expectativas de la sociedad civil o los tomadores de decisión. Esto es relevante, ya que a la vista de las políticas globales y guías internacionales (IFRC, 2015; UNASUR, 2015; UNISDR, 2015) para reducir el riesgo de desastres (RRD), fortalecer la institucionalidad y gobernanza es crucial.

Lo anterior será pues el hilo conductor de esta investigación que utilizó a Chile como caso de estudio luego del Terremoto del 27 de febrero de 2010 -conocido popularmente como 27Fque causó más de 500 muertes y daños cercanos al 18\% del Producto Interno Bruto (PIB). Esta país sudamericano fue seleccionado también por su perfil multi-amenaza (Kappes, Keiler, von Elverfeldt, \& Glade, 2012; PreventionWeb, 2016), la susceptibilidad que posee frente a riesgos intensivos como extensivos (UNISDR, 2009) y la carencia hasta el día de hoy de una ley relativa a la gestión del riesgo (BID, 2015; PreventionWeb, 2016). Dados estos antecedentes, se consideró un caso de estudio apropiado para analizar cómo las PPD evolucionan y caracterizar el supuesto cambio que esta catástrofe produjo. Además, se ha tenido a la vista que en la mayoría de los casos, la investigación realizada sobre cambio de políticas públicas y desastres se ha concentrado en los Estados Unidos de América (Birkland, 1997, 2006; Birkmann et al., 2010) por lo que emplear un caso de estudio, en una región distinta del planeta, provee una nueva mirada al proceso y amplía la incipiente investigación en el área.

Este artículo comenzará enmarcando teóricamente el proceso de cambio de políticas públicas en el enfoque de las corrientes múltiples (Kingdon, 1995) para luego describir la metodología y los resultados principales de la investigación. Posteriormente se derivará en una discusión sobre estado y cambio en las PPD en Chile, así como sus brechas y propuestas para mejorar la introducción de cambio con transformación en las políticas. Este articulo concluye determinando el alcance y la profundidad del cambio en Chile post-27F.

\section{CAMBIO Y POLÍTICAS PÚBLICAS DE DESASTRES}

La forma en que una crisis es comprendida puede diferir dependiendo desde el punto de vista que se le mire; un desastre es para una vasta mayoría un fenómeno negativo, aunque también es cierto que cada crisis trae una oportunidad. Por ende, se puede establecer que estos eventos tienen un carácter dual y ambiguo. Desde una aproximación positiva o emancipadora como Beck (2015) plantea; los males, producen bienes comunes, es decir, podemos argumentar que luego de un desastre se puede abrir una ventana de oportunidad para introducir cambio positivo cuando, por ejemplo, las políticas fallan. En este sentido, las catástrofes se transforman en un evento focalizador (Birkland, 1997), una oportunidad para que legisladores, tomadores de decisión, emprendedores de política pública (P. Sabatier, 2007) y la sociedad civil organizada puedan centrar la atención en las políticas orientadas a reducir el riesgo de desastre para introducir soluciones en aspectos tales como la mitigación o el fortalecimiento de las capacidades de respuesta. 
De ser cierto lo anterior, también es necesario comprender la extensión de las supuestas mejoras que este fenómeno conlleva analizando la lógica del sistema en cuanto a cómo las instituciones nacionales y autoridades conciben las acciones para gestionar el riesgo de desastre, pues no todo cambio de por si es positivo. Por ende, la valoración y profundidad de una variación en cuanto esta genere transformación, debe ser analizada para determinar si produce una metamorfosis (Beck, 2015) en la lógica del sistema. Es decir, el cambio de PPD puede concebirse no sólo como las acciones dirigidas a evitar la anterior catástrofe, sino como una oportunidad para proyectarse hacia el futuro.

Sin embargo, la aproximación común al cambio de PPD es desde una postura retrospectiva como lo plantea Birkland (1997): el cambio de políticas públicas está orientado a responder al último desastre y no a los futuros. Más aún, la percepción y conciencia del riesgo está determinada en gran parte por haber experimentado una catástrofe (Wachinger, Renn, Begg, \& Kuhlicke, 2013). Por esto, es de vital importancia emplear la oportunidad que trae una crisis y revisar el sistema de manera integral y prospectiva para mejorar transversalmente las políticas públicas orientadas a reducir el riesgo de desastre.

Pues bien, para comprender el cambio de políticas públicas existe más de una teoría ( $P$. Sabatier, 2007) en la disciplina que ha desarrollado la investigación en esta área, la ciencia política. Esta investigación empleó el enfoque de las corrientes múltiples (Kingdon, 1995) debido a su capacidad de explicar cómo las políticas de un gobierno son realizadas en condiciones de ambigüedad (Zahariadis, 2007) y; si entendemos al desastre como un constructo social, esto tienen una complejidad y ambigüedad inherente (Pidgeon \& O'Leary, 2000) que exige utilizar métodos comprensivos para analizarlo, tal como el de las corrientes múltiples. Por lo demás, este enfoque ha sido probado en un rango de contextos y niveles, poseyendo una riqueza empírica sin paralelos (Jones et al., 2016).

\section{El Enfoque de las Corrientes Múltiples}

El enfoque de las corrientes múltiples planteado por Kingdon (1995) estima que dentro de los gobiernos y la arena de las políticas públicas existen tres corrientes: problemas, políticas y política. Estas, al ocurrir un evento focalizador como un desastre, son acopladas por los emprendedores de política pública para generar cambio aprovechando la apertura de la ventana de oportunidad. En la Figura 1 se observa un diagrama con las corrientes y sus subcomponentes, las que serán detalladas a continuación

En cuanto a la corriente de los problemas, esta comprende la serie de asuntos públicos que los legisladores, tomadores de decisión y los ciudadanos quieren que sean considerados (Jones et al., 2016). En el caso post-desastre, la falla en los instrumentos de gestión como las políticas o una pobre gestión de la emergencia se pueden convertir en problemas que ingresan a la corriente. En este sentido, la atención puede provenir no sólo de un evento focalizador sino también de los indicadores o retroalimentación del sistema.

La corriente de políticas es una colección finita, pero compleja de ideas y posibilidades para resolver un problema según Jones et al. (2016). Así, es más probable que después y no antes de una catástrofe, surjan diversas opiniones sobre cómo mejorar las políticas públicas de gestión del riesgo de desastre. Este fenómeno se explica en los subcomponentes de la corriente de políticas, en particular la viabilidad técnica y la aceptabilidad de valor (Kingdon, 1995), ya que las medidas para reducir el riesgo ocurren en circunstancias económicas y políticas determinadas (Wisner, 2003). Ello, sumado a cortos periodos presidenciales ${ }^{2}$, fomenta un mayor apetito al riesgo y desincentiva inversiones para construir resiliencia (Overseas Development Institute \& The World Bank, 2015).

La política por otro lado, es una corriente que se compone de tres elementos: el ánimo nacional, los grupos de presión y los cambios en la administración. Es decir, para que una crisis tenga el potencial de generar cambio en las PPD por ejemplo; se requieren suficientes recursos para influenciar el discurso político y atraer la atención de la élites (Stallings, 1991). Por ende, la temática en torno a los desastres podría parecer destinada a ser un asunto ex-post. Por último, dentro de

2. Chile tiene un periodo presiden cial de cuatro años, sin reelección (República de Chile, 1980). 
la corriente política, es relevante destacar que según Zahariadis (2007) la combinación de un ambiente nacional favorable a modificaciones junto con un cambio en la administración del Estado, es lo que ejerce el mayor efecto en las agendas, combinación que existía en Chile ya que el cambio de gobierno fue en menos de un mes, después del $27 \mathrm{~F}$.

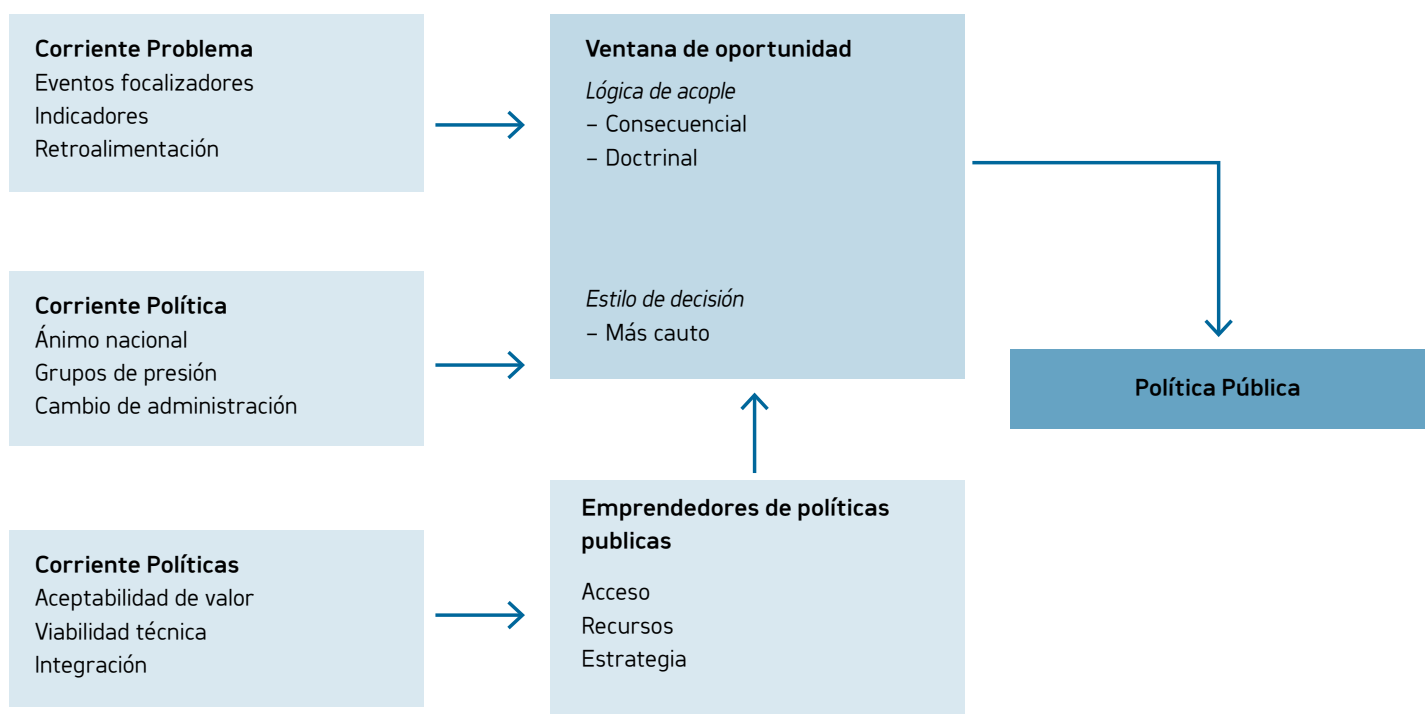

Figura 1: Diagrama del enfoque de las corrientes múltiples. Fuente: Autor, adaptado de Zahariadis (2007, p. 71)

\section{Cambio}

Hemos hablado de cambio, pero no lo hemos conceptualizado, mas no nos detendremos en analizar la complejidad de su definición. Lo que si haremos es categorizarlo en dos términos (Beck, 2015, p. 63): cambio social por un lado; entendido como la reproducción del orden social y político, y metamorfosis por el otro; que es la transfiguración de ese orden social y político. Con esto queremos decir que si deseamos generar un cambio en la lógica de un sistema que busca gestionar el riesgo de desastres, debemos apuntar a una transfiguración o transformación del mismo, enfrentando como propone Manyena (2013) la falta de poder de la agencia a cargo de RRD, marcos institucionales y legales inadecuados, y la posible falla de integración de la Gestión del Riesgo al desarrollo.

\section{MÉTODOS}

El cambio en las políticas públicas relacionadas a la GRD en esta investigación empleó un enfoque cualitativo como Phillips (2002) sugiere, utilizando las siguientes fuentes secundarias: leyes, regulaciones y presupuestos relacionados directamente a la Gestión del Riesgo de desastres y Protección Civil en Chile, omitiendo ordenanzas, códigos, y regulaciones sectoriales debido a las limitaciones de tiempo y presupuesto que planteaba la investigación. Sobre este último, se analizó exclusivamente el de la Oficina Nacional de Emergencias del Ministerio del Interior y Seguridad Pública (ONEMI) en los últimos diez años; pues se asume que el cambio de política pública es apreciable luego de un decenio (P. A. Sabatier \& Jenkins-Smith, 1993). Los montos fueron obtenidos de las leyes de presupuesto anual del Estado de Chile desde el año 2006 al 2016 y corregidos según el Índice de Precios del Consumidor (IPC) al mes de promulgación de la ley de presupuesto respectiva con el presupuesto del 2016.

El actual decreto que establece el Plan Nacional de Protección Civil (República de Chile, 2002), la Política Nacional para la Gestión del Riesgo de Desastres (ONEMI, 2014), y el actual proyecto de ley que establece el Sistema Nacional de Emergencia y Protección Civil y crea la Agencia Nacional de Protección Civil Boletín 7550-06 (Gobierno de Chile, 2011); fueron analizados a través de un conteo de palabras con el software NVivo, aplicando el siguiente criterio: cinco letras mínimo de extensión, las diez palabras más frecuentes y se incluyeron sinónimos. Teniendo en cuenta lo anterior, el propósito del método fue descubrir los conceptos predominantes en los marcos normativos y 
encontrar su enfoque. Además, se realizó un análisis comparativo entre los documentos nombrados para observar la variación en sus términos.

Esta investigación también utilizó fuentes primarias en la forma de entrevistas semi-estructuradas a representantes de cinco grupos de interés: gobierno, legisladores, sociedad civil organizada, medios y la academia. Se solicitaron 35 entrevistas anónimas, pero sólo 19 entrevistados accedieron a ellas. El cuestionario fue diseñado para determinar si una ventana de oportunidad se abrió luego del $27 \mathrm{~F}$ y, de haberlo hecho, de qué manera se consolidó. Además, las entrevistas exploraron cómo los grupos de interés obtuvieron sus lecciones aprendidas o por aprender luego del desastre, cuáles fueron estas lecciones y, finalmente, si han implementado o diseñado PPD a partir de ellas. Asimismo, las entrevistas indagaron la percepción del cambio en las PPD antes o después de la catástrofe. Por último, se sondeó la opinión respecto al rol de los medios de comunicación en la RRD y cuán participativo ha sido el diseño de PPD en Chile.

Se diseñó un sistema de codificación para establecer el anonimato entre los entrevistados y fomentar la honestidad en las respuestas. Este código cuenta con tres secciones que derivan en el siguiente formato: AA-BB-CC, el que no será detallado para asegurar la confidencialidad.

Los siguientes individuos fueron entrevistados por categoría:

- Gobierno

- ONEMI:

\section{Director}

Sub-director

Sub-director de Gestión del Riesgo

También se seleccionaron ministerios que integraran el Comité Nacional de Protección Civil y el Comité Nacional de Operaciones de Emergencia (República de Chile, 2002). Los siguientes accedieron a la entrevista a través de su encargado o representante en GRD:

- Ministerio de Defensa Nacional

- Ministerio de Energía

- Ministerio de Vivienda y Urbanismo

- Legisladores

Se seleccionaron los Senadores y Diputados que integraban la Comisión de Gobierno, Regionalización y Descentralización tanto de la Cámara de Diputados como en el Senado. Esta decisión se basa en que esta comisión ha sido designada como la principal para tratar los proyectos de ley relacionados a la GRD. Trece miembros componen la comisión en la Cámara de Diputados y cinco en el Senado. Sólo 8 accedieron a ser entrevistados.

- Sociedad Civil Organizada

La investigación descubrió tres organizaciones relevantes, pero sólo dos accedieron a ser entrevistadas:

- $\quad$ Red de Ayuda Humanitaria de Chile (RaHCH)

- $\quad$ Red Emergencia

- Medios

Se contactó a 5 periodistas ancla de los principales noticieros del país. Sólo 2 accedieron una entrevista.

- Academia

De las tres organizaciones académicas ligadas a la RRD, sólo REDULAC fue entrevistada. 


\section{RESULTADOS}

\section{Fuentes Secundarias}

Ahora bien, en el caso del presupuesto de ONEMI la Figura 2 demuestra que desde el año 2006 ha tendido a aumentar, a pesar del declive en los últimos periodos. Sin embargo, al observar con mayor detalle las asignaciones monetarias se verifica que una parte relevante del presupuesto va directamente a financiar la red sismológica, que es dirigida por la Facultad de Ciencias Físicas y Matemáticas de la Universidad de Chile. Por ejemplo, el año 2010 el presupuesto total de ONEMI eran CLP\$8.914.510.000, pero casi la mitad de este monto fue destinado a la red sismológica. En cuanto a fondos para educación a la comunidad, su gasto promedio durante los años 2006-2016 en programas fue de CLP\$184.212.000 con una desviación estándar de $\$ 68.211 .000$ pesos chilenos.

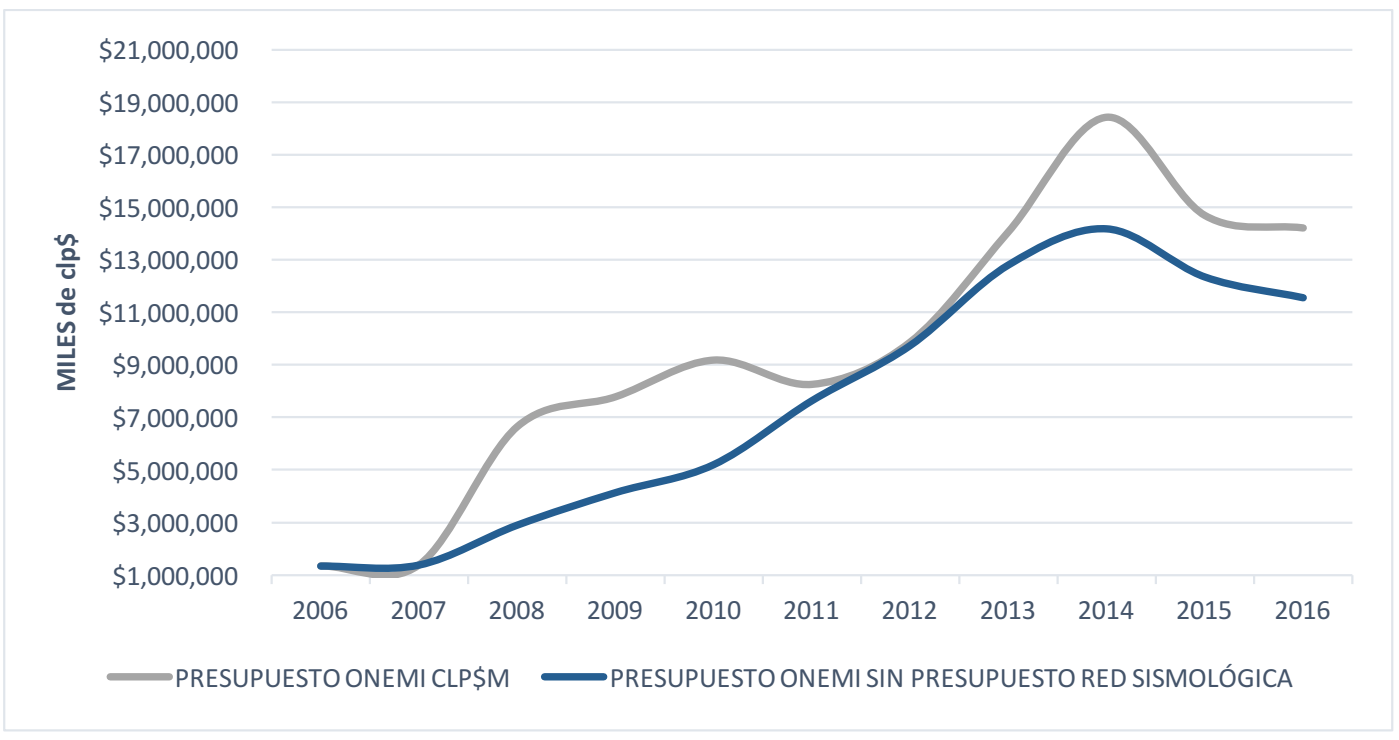

Figura 2: Evolución Presupuesto Onemi 2006-2016. Fuente: Autor, 2018

Respecto a la revisión instrumentos normativos; luego de un conteo de palabras del actual decreto que establece el Plan Nacional de Protección Civil, la investigación descubrió que los tres términos predominantes son: emergencia, reporte y protección; conectado probablemente a este último término está el cuarto; civil formando el concepto de protección civil.

La investigación realizó un análisis comparativo de las diez palabras más frecuentes entre el actual Plan Nacional de Protección Civil y el proyecto de ley Boletín 7550-06 en la Tabla 2. Los resultados demuestran una importante variación en los términos, teniendo sólo en común emergencia destacado en azul. También se observa un cambio hacia el riesgo, siendo la segunda palabra predominante en el actual proyecto de ley, que se encuentra conectada probablemente con la tercera gestión, formando el término gestión de riesgo.

En cuanto a la actual Política Nacional para la Gestión del Riesgo de Desastres, se realizó el mismo conteo de palabras y se comparó con los otros dos documentos normativos como se observa en la Tabla 3, en donde los colores de las celdas indican similitud en los términos. Hay pues un alineamiento en la terminología en los instrumentos generados post-desastre del 2010 y una clara diferencia con el Plan Nacional de Protección Civil del año 2002. 


\begin{tabular}{ccc}
\hline & Plan Nacional de Protección Civil & \\
\hline Palabra & $\mathbf{N}^{\mathbf{0}}$ & Palabras similares \\
emergencia & 328 & emergencia, emergencias \\
informe & 148 & informe, informes \\
protección & 145 & Protección \\
civil & 142 & Civil \\
evento & 137 & evento, eventos \\
nivel & 128 & nivel, niveles \\
daños & 121 & Daños \\
recursos & 114 & recurso, recursos \\
información & 108 & información \\
desastre & 103 & desastre, desastres \\
\hline
\end{tabular}

Tabla 1: Diez primeras palabras predominantes del Plan Nacional de Protección Civil.

Fuente: Autor, 2018

\begin{tabular}{cccc}
\hline \multicolumn{2}{c}{ Plan Nacional de Protección Civil } & \multicolumn{2}{c}{ Proyecto de ley Boletín 7550-06 } \\
\hline Palabra & $\mathbf{N}^{\circ}$ & Palabra & $\mathbf{N}^{\mathbf{0}}$ \\
\hline emergencia & 328 & emergencias & 358 \\
informe & 148 & riesgos & 221 \\
protección & 145 & gestión & 220 \\
civil & 142 & nacional & 203 \\
evento & 137 & servicio & 125 \\
nivel & 128 & comité & 89 \\
daños & 121 & sistema & 89 \\
recursos & 114 & provincial & 82 \\
información & 108 & política & 77 \\
desastre & 103 & ministros & 63 \\
\hline
\end{tabular}

Tabla 2: Comparado entre las diez palabras predominantes del Plan Nacional de Protección Civil y el proyecto de ley Boletín 7550-06.

Fuente: Autor, 2018

\begin{tabular}{|c|c|c|c|c|c|}
\hline \multicolumn{2}{|c|}{ Plan Nacional de Protección Civil } & \multicolumn{2}{|c|}{ Proyecto de Ley Boletín 7550-06 } & \multicolumn{2}{|c|}{$\begin{array}{c}\text { Política Nacional para la Gestión del Riesgo de } \\
\text { Desastre }\end{array}$} \\
\hline Palabra & $\mathbf{N}^{\circ}$ & Palabra & $\mathbf{N}^{\circ}$ & Palabra & $\mathbf{N}^{\circ}$ \\
\hline emergencia & 328 & emergencia & 358 & desastres & 166 \\
\hline informe & 148 & riesgos & 221 & riesgo & 166 \\
\hline protección & 145 & gestión & 220 & nacional & 144 \\
\hline civil & 142 & nacional & 203 & gestión & 94 \\
\hline evento & 137 & servicio & 125 & política & 92 \\
\hline nivel & 128 & comité & 89 & reducción & 54 \\
\hline daños & 121 & sistema & 89 & emergencia & 48 \\
\hline recursos & 114 & provincial & 82 & amenazas & 46 \\
\hline información & 108 & política & 77 & ministerio & 46 \\
\hline desastre & 103 & ministros & 63 & desarrollo & 44 \\
\hline
\end{tabular}

Tabla 3: Comparado entre las diez palabras predominantes del Plan Nacional de Protección Civil, el proyecto de ley Boletín 7550-06 y la Política Nacional para la Gestión del Riesgo de Desastre.

Fuente: Autor, 2018 


\section{Entrevistas}

A continuación, presentaremos los principales resultados que arrojaron las entrevistas por pregunta.

P1. ¿Cuáles son los tres desastres de mayor relevancia que usted recuerda?

\section{7 entrevistados respondieron.}

Los desastres más recordados son aquellos originados por terremotos (Ver Tabla 4), con una predominancia del evento ocurrido en 2010 . En segundo lugar, los aluviones, particularmente los ocurridos en el norte de Chile el año 2015 y, finalmente, erupciones volcánicas junto con incendios forestales.

\begin{tabular}{ccc}
\hline Palabra & No $^{\circ}$ & Palabras Similares \\
\hline terremoto & 50 & terremoto, terremotos \\
desastre & 21 & desastre, desastres \\
emergencia & 17 & emergencia, emergencias \\
aluviones & 14 & Aluviones \\
atacama & 12 & Atacama \\
región & 12 & Región \\
estado & 10 & Estado \\
erupción & 8 & Erupción \\
incendio & 8 & incendio, incendios \\
iquique & 8 & Iquique \\
\hline
\end{tabular}

Tabla 4: Desastres más relevantes y recordados. Fuente: Autor, 2018

P2. ¿Cuál es su opinión sobre el marco institucional vigente en Chile para reducir el riesgo de desastres?

19 entrevistados respondieron.

Existe mayoritariamente, como lo demuestra la Figura 3, una opinión negativa respecto al marco institucional vigente en Chile para Reducción de Riesgo de Desastres. Reactivo, centralizado, inadecuado y limitado son algunos de los términos que los entrevistados emplearon para describirlo. Sólo un individuo tuvo una opinión positiva.

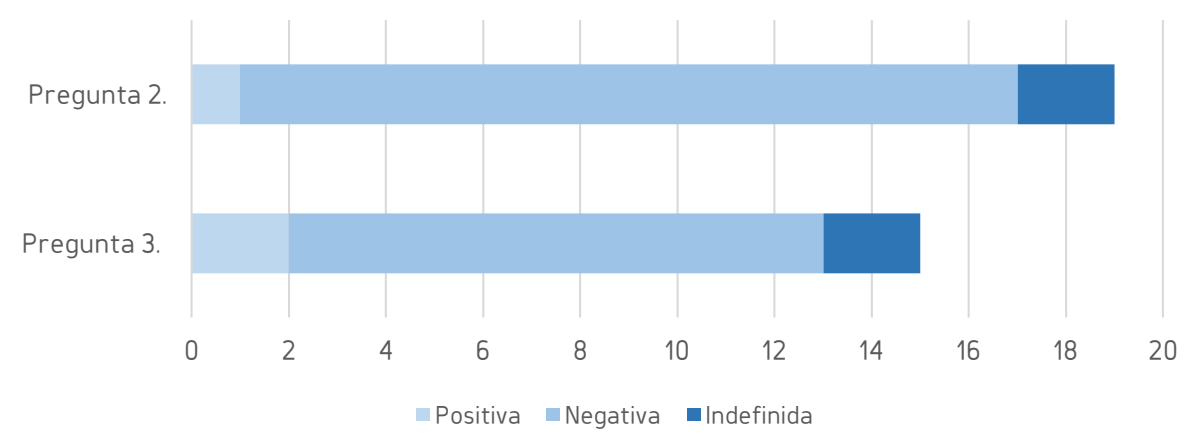

Figura 3: Opiniones respecto al marco institucional vigente y presupuesto para RRD. Fuente: Autor, 2018

P3. ¿Cuál es su opinión sobre el presupuesto vigente en Chile para reducir el riesgo de desastres?

15 entrevistados respondieron.

Al igual que en la pregunta 2, la mayoría de los entrevistados (Ver Figura 3) estiman que la asignación presupuestaria para RRD es insuficiente. Algunas respuestas revelan que los individuos estiman que la fase de respuesta se encuentra bien financiada, pero que las acciones preventivas carecen de un presupuesto adecuado. 
P4. ¿Cree que se abrió una ventana de oportunidad en Chile después del terremoto del 27 de Febrero de 2010?

19 entrevistados respondieron.

Todos los entrevistados, salvo uno, tuvieron una respuesta positiva a la apertura de una ventana de oportunidad luego del 27F. Como GO-MD-01, quien declaró que el evento "abrió una puerta, ahora están todos interesados (en RRD)" y abrió "oportunidades de mejoras sustanciales" acorde a GO-ON-02. Es interesante que algunos entrevistados como LE-SE-03 y GO-MV-01 elevaron la pregunta sobre por cuánto tiempo permanece abierta la ventana de oportunidad.

P5. ¿Qué lecciones a aprender o aprendidas emergieron después del terremoto del 27 de Febrero de 2010?

11 entrevistados respondieron.

A continuación, se presentarán las principales lecciones por entrevistado.

SC-RA-01

- Mejorar los métodos de evaluación de daños

- Coordinación

SC-RE-01

- Un sistema centralizado es inútil

- Las comunicaciones no pueden perderse durante una emergencia

GO-MD-01

- Es necesario un trabajo cooperativo

- Modificar los protocolos y normativas vigentes

- Incorporar a las Fuerzas Armadas en el sistema de protección civil

GO-ON-01

- Mejorar las capacidades de respuesta

- Actuar con mayor aversión al riesgo

- Hay un vacío institucional

GO-ON-02

- El país necesita una normativa para gestionar el riesgo de desastre

- Mejorar la estructura organizacional de los Comités de Operaciones de Emergencia

- La reducción del riesgo de desastre debe ser una política de Estado

GO-ON-03

- Después de cada catástrofe, Chile genera cambio institucional

- Mejor conocimiento de la amenaza de tsunami

- Se requiere mayor tiempo para comprender el terremoto del 2010 y aprender lecciones

- Chile tiene códigos de construcción de clase mundial

LE-DI-04

- Los ciudadanos son más conscientes del riesgo

- Se necesita que la planificación urbana considere las zonas de amenaza 
LE-DI-11

- Cada terremoto es una lección, mas ya no es tiempo de seguir aprendiendo. Las lecciones ya se debieran haber aprendido

- Se requiere desarrollar un fondo monetario para ayudar a la gente afectada

LE-SE-02

- El país no estaba preparado para cualquier evento

- Chile tiene códigos de construcción apropiados

- Nada o muy poco se ha aprendido

LE-SE-03

- Se requiere un nuevo marco institucional para enfrentar desastres

- Mejorar los sistemas de comunicación de emergencia

LE-SE-05

- Fallas en los sistemas de comunicaciones.

- Necesidad de mejorar el proceso de toma de decisión frente a una crisis.

P6. ¿Cuál es su opinión respecto a la reducción del riesgo de desastres en Chile como prioridad nacional en los últimos 10 años?

6 entrevistados respondieron.

Un enfoque evolucionario o de equilibrio puntuado (True, Jones, \& Baumgartner, 2007) predomina en la percepción alrededor de la RRD en Chile. Todos los respondientes reconocen un cambio durante la última década. También reconocen el efecto que tuvo el $27 \mathrm{~F}$ en producir dicho cambio. Sin embargo, un legislador hizo presente el problema de cómo el interés en ocuparse de los desastres se difumina luego de la atención inicial que conlleva una catástrofe.

P7. ¿Cuál es su opinión sobre el rol de los medios de prensa en la reducción del riesgo de desastres?

\section{7 entrevistados respondieron.}

Las entrevistas revelan que la mayoría -9- de los entrevistados consideran el rol de los medios casi exclusivamente como reportar el desastre y critican el enfoque sensacionalista que ellos tienen. Se espera de los medios masivos que sean un instrumento para transmitir información sobre cómo responder a la emergencia presente y describir la situación. Tres consultados propusieron otros roles que los medios debieran tener en cuanto a investigar las causas del desastre o educar en prevención.

P8. ¿Cuál es su opinión sobre la participación ciudadana en las actuales iniciativas de política pública de desastres?

\section{9 entrevistados respondieron.}

Sobre la participación en el diseño PPD no hay consenso entre los entrevistados. Como la Figura 4 indica, mientras los legisladores consideran como participativo el proceso; el resto de los grupos de interés tiene una opinión negativa al respecto, sintiéndose excluidos del proceso en algunos casos, incluso representantes de la ONEMI. 


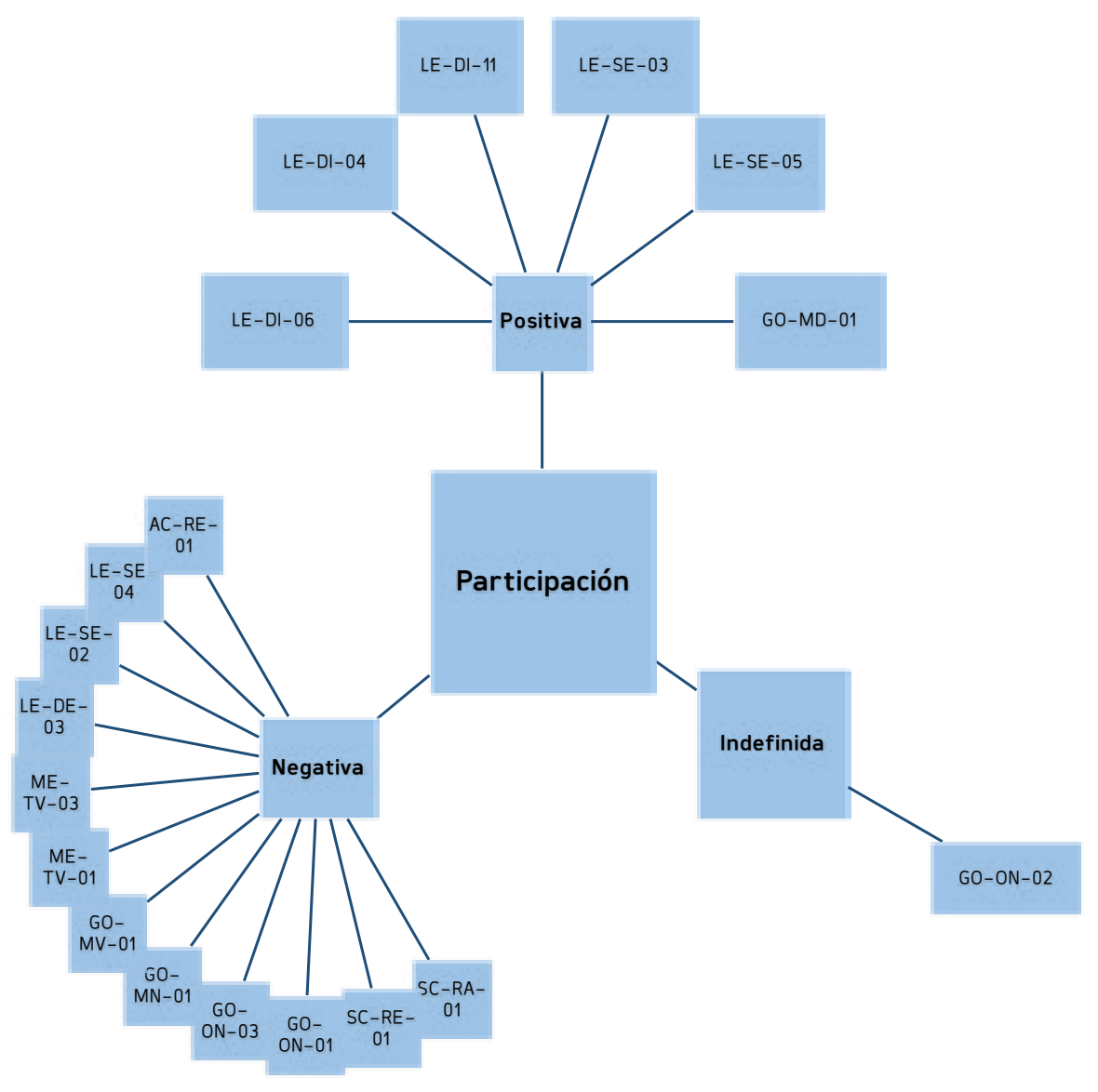

Figura 4: Percepción de la participación en el diseño de PPD. Fuente: Autor, 2018

\section{DISCUSIÓN}

Hasta el momento hemos analizado los cuerpos normativos vigentes y prospectivos relativos a la RRD y la protección civil en Chile. Asimismo, se han examinado las asignaciones presupuestarias de ONEMI y se han realizado entrevistas a 19 individuos representado a 5 grupos de interés. El propósito fue identificar los elementos que componen el cambio de política de acuerdo al enfoque de las corrientes múltiples y, explorar la percepción alrededor de la RRD y la participación en el diseño de PPD en Chile.

Seis años luego del desastre del 27F los resultados de esta investigación revelan que una ventana de oportunidad se abrió para introducir cambio en las políticas públicas de desastre en Chile a nivel nacional. El hecho que el Terremoto del 2010 sea el evento más recordado entre los entrevistados sustenta la hipótesis de que esta crisis tuvo la capacidad de atraer y mantener la atención hacia el sistema de protección civil. Por ende, podemos concluir que esta catástrofe fue un evento focalizador, capaz de abrir una ventana de oportunidad para revisar e introducir cambio principalmente en las capacidades de respuesta y preparación del sistema.

Un ejemplo de lo anterior fue la creación de una comisión investigadora en la Cámara de Diputados a menos de dos semanas de ocurrido el terremoto. El rol de esta comisión fue examinar el estado del marco institucional para responder a desastres y evaluar mejoras tecnológicas (Cámara de Diputados de la República de Chile, 2012). La principal recomendación de esta instancia fue que el Estado de Chile debía implementar políticas públicas orientadas a mejorar la respuesta ante catástrofes. Lo anterior demuestra que el foco de los legisladores continuó en las capacidades de respuesta y no en medidas para reducir el riesgo de desastres como la pobreza multi-dimensional que algunos estudios (Sanhueza, Contreras, \& Denis, 2012) indican, aumentó en las zonas afectadas por la catástrofe. Aún más, uno de los legisladores entrevistados indicó que la ventana de oportunidad sólo se abrió desde un "punto de vista declarativo." 
¿El resultado? Si se observa el Índice de Gobernabilidad y Políticas Públicas en Gestión del Riesgo de Desastres (iGOPP) del Banco Interamericano de Desarrollo (2015), el componente preparativos para la respuesta alcanza el mayor nivel de cumplimiento con un $44 \%$, pero el iGOPP revela que aún Chile se encuentra en un nivel incipiente, con sólo un 28,57\%. Es decir, la ocasión para el cambio se focalizó en los requerimientos de equipamiento para responder a emergencias como el robustecimiento del sistema de comunicaciones o el mantenimiento de la continuidad operacional al construir de un moderno edificio con disipadores sísmicos para la oficina central de ONEMI, mas no para el capital humano como SC-RE-01 indicó.

Ahora bien, los resultados del análisis de los instrumentos normativos arrojaron resultados interesantes. Por un lado, se observó un cambio en los conceptos empleados en la Política Nacional de GRD y el proyecto de ley, existiendo mayor énfasis en la gestión del riesgo en comparación al actual Plan Nacional de Protección Civil del año 2002. Sin embargo, la centralización se mantiene como un asunto recurrente. Por ejemplo, la indicación sustitutiva presentada al actual proyecto de ley Boletín 7550-05 no incluía en el sistema nacional de gestión de riesgos y emergencias al nivel municipal. Así mismo, a pesar de que el proyecto indica que el nuevo servicio es desconcentrado en el territorio, también dice que es centralizado, dependiendo directamente del Ministerio del Interior y Seguridad Pública. Desde la perspectiva de administración del estado, esta composición -institución desconcentrada y centralizada- no subsana el centralismo que el representante de la academia $\mathrm{AC}-\mathrm{RE}-01$ indica debiera corregirse otorgándole a ONEMI recursos propios para no depender del poder político.

A partir de los componentes del enfoque de las corrientes múltiples, el problema está circunscrito a las brechas e inconvenientes que emergieron luego del Terremoto del 2010, desde una mirada retrospectiva. Los resultados de la investigación demuestran que la mayoría de los entrevistados tienen una opinión negativa de las condiciones previas al $27 \mathrm{~F}$ y la respuesta al evento en cuanto a las capacidades que se esperaba que tuviera el sistema para responder a la emergencia. Los individuos consultados también reconocen que aún el marco institucional y el presupuesto para gestionar y reducir el riesgo de desastre es inadecuado, lo que es coherente con lo que indican varios reportes e indicadores (Banco Interamericano de Desarollo, 2015; DIPECHO, 2012; Gobierno de Chile, 2013) sobre el estado del arte de la RRD en Chile.

Sin embargo, a pesar de que luego del Terremoto del 2010, el gobierno de Chile presentó el proyecto de ley que establece el Sistema Nacional de Emergencia y Protección Civil y crea la Agencia Nacional de Protección Civil, Boletín 7550-06, su aproximación siguió siendo orientada hacia la respuesta, probablemente por la comprensión que el sistema legislativo y los asesores tenían del problema y sus soluciones. Sumado a esto, la investigación descubrió un bajo nivel de participación ciudadana en el diseño y tramitación de las PPD. La mayoría de los entrevistados expresaron que se sintieron excluidos, incluso marginados, como declaró SC-RE-01 del proceso. El representante de la RAHCh indicó que le han "perdido el rastro a en que va la ley" [Sic] y denuncian la falta de una "construcción conjunta." No obstante, es interesante destacar que la mirada disidente a lo anterior proviene desde el gobierno y los legisladores, como GO-MD-01 quien expresó que "esta política pública, notoriamente, ha tenido una participación mayor."

Se podría suponer que la falta de participación se debe a la ausencia de la temática de gestión de riesgo en la agenda. En este sentido, el haber explorado la percepción sobre los medios, quienes son la institución primaria para fijar la agenda (McCombs \& Shaw, 1972), permitió descubrir que gran parte de los entrevistados comprende el rol de la prensa desde la cobertura del desastre, transmitir los requerimientos de las autoridades en tiempos de crisis y, sólo unos pocos individuos, sondean la posibilidad de que los medios sean un elemento crucial para indagar las fallas y vulnerabilidades que determinaron la ocurrencia del desastre. Con todo, si tenemos presente la lista de funciones que los medios puede ejercer en la RDD según Olson, Sarmiento Prieto \& Hoberman (2010), al parecer se está perdiendo la oportunidad de emplear a este grupo de interés o cuarto poder para impulsar la reducción del riesgo y fomentar una cultura preventiva.

Por otra parte, cuando analizamos los presupuestos de ONEMI, a pesar de observar una 
tendencia al crecimiento, su monto es modesto a la luz de los desafíos que tiene el actual sistema chileno de protección civil. Según las entrevistas, tan altos han sido los costos de los desastres en Chile en los últimos años, que su financiamiento se sostiene casi exclusivamente en fondos de emergencia extraordinarios, sin mayor control generando controversias que levantó la prensa chilena (Revista Capital, 2016). En este sentido, Chile es el país con el mayor daño promedio en su PIB dentro de los países de OCDE entre los años 1980 al 2011 (OECD, 2012). Aún más, uno de los representantes de ONEMI indicó en la entrevista que el impacto de los desastres el 2015 fue tan alto que afectó el desarrollo del programa presidencial de gobierno.

En vista de lo anterior, una de las recomendaciones principales de las políticas globales, es invertir en programas educacionales para reducir el riesgo de desastre. Acorde a uno de los directivos de ONEMI "se ha hecho bastante al menos en preparación ciudadana". Sin embargo, como los resultados de la investigación demostraron, sólo un $2 \%$ del presupuesto promedio de esta oficina se ha destinado a programas de entrenamiento en protección civil. Vale destacar, que la muestra está dispersa, pues el año 2016 se duplicó el presupuesto en esta área. Empero, la desviación estándar del periodo 2006-2015 es de CLP\$37.030.000 con un promedio de $\$ 166.583 .000$ pesos chilenos. Por ende, no ha habido una variación relevante en este punto.

En cuanto al cambio institucional propuesto en el proyecto de ley; elevar la jerarquía administrativa (República de Chile, 2000) de ONEMI de una oficina, el nivel más bajo, a un nivel medio de agencia o servicio eleva dudas respecto a la capacidad que tendría esta nueva institución para coordinar el sistema. En este sentido, Mena (2014) observó además que el proyecto de ley mantiene su foco en las fases de preparación y respuesta, sin abarcar todas las fases de la gestión del riesgo de desastre.

Considerando lo anterior, es crítico a analizar las expectativas que tienen los grupos de interés en el proyecto de ley Boletín 7550-06, tanto en su capacidad de implementar como de sostener y reforzar las acciones necesarias para gestionar y reducir el riesgo de desastres. Sin embargo, incluso los legisladores reconocen como insuficiente el actual proyecto. Uno de los Senadores entrevistados indicó respecto a la tramitación de la ley: "votamos ahora en el Senado esta nueva institución, pero todos los discursos dijeron mire, no es lo que queremos, no es lo que necesitamos. Así fueron todos los discursos, pero bueno, fue lo que votamos; entonces, si tú me preguntas ¿qué es lo aprendido? Yo creo que desgraciadamente poco, muy poco." A pesar de lo anterior, el proyecto se aprobó en general en el Senado de Chile. Se observa pues, una falta de alineación entre las soluciones y los problemas. Las causas se pueden encontrar, a la luz de esta investigación, en la falta de participación en el diseño y una carencia de emprendedores de PPD, obteniendo un cambio modesto en ellas, como el presupuesto de ONEMI.

Entonces, al parecer, los problemas y la falta de soluciones apropiadas a ellos en forma de políticas públicas nos lleva a prestar mayor atención a un elemento del enfoque de las corrientes múltiples, el emprendedor de políticas públicas. El rol de este individuo o actor corporativo, es acoplar las soluciones que existen dentro, y más frecuentemente, fuera del ambiente legislativo con los problemas en un contexto político particular. Sin embargo, la investigación revela que no existe una organización o individuos que sean reconocidos como emprendedores de política pública, un rol que consideramos ha sido subestimado en el contexto chileno para producir no sólo un cambio, sino una metamorfosis en la manera de abordar la gestión del riesgo de desastre. Como hemos observado, el proceso de diseño y cambio de PPD en Chile es selectivo y desfavorece la participación. Mas, como Zahariadis (2007, p. 74) indica "when windows open, policy entrepreneurs must immediately seize the opportunity to initiate action. Otherwise, the opportunity is lost."

Sin embargo, para lograr lo anterior, los emprendedores de política pública necesitan acceso a la política y a los legisladores. Empero, la investigación reveló que incluso organizaciones de las cuales se espera que sean emprendedores, como ONEMI, expresaron en las entrevistas que " (ONEMI) no fue nunca tomada en cuenta" en el diseño del proyecto de ley vigente. Por lo tanto, una recomendación que extrae esta investigación para este y otros procesos de cambio en PPD post-desastre, es fomentar y reconocer la existencia de emprendedores de política pública. De 
esta manera, las oportunidades que traen los eventos focalizadores como los desastres, puede ser tomados para impulsar la metamorfosis y crear cambio con verdadera transformación.

\section{Espacios de mejora}

Considerando los resultados de la investigación y la discusión anterior, encontramos apropiado explorar algunas acciones para mejorar el cambio de políticas públicas de desastres.

Pues bien, esta investigación descubrió que aún existen problemas, en términos del enfoque de corrientes múltiples, en el sistema de protección civil chileno. Una opinión negativa por parte de los grupos de interés del marco institucional vigente y el presupuesto, acoplado a las fallas y vulnerabilidades que el terremoto del 2010 expuso, impulsó un cambio gracias a la ventana de oportunidad generada por este evento. Sin embargo, las soluciones o políticas públicas disponibles para corregir el sistema tienen valores que aún centran las acciones en la reacción a emergencias, al igual que las lecciones extraídas del desastre. Lo último, unido a un proceso con pobre participación derivó en un cambio sin la capacidad de generar transformación en la lógica del sistema.

En vista de lo anterior, se aprecia que es fundamental desarrollar y apoyar un proceso participativo real. Esto permite, en primer lugar, incluir más visiones sobre las falencias y problemas identificados ex-post. De la misma manera, el permitir que varios grupos de interés puedan poner sobre la mesa soluciones, amplía el rango de estos y hace más holístico el proceso de cambio. Aún más relevante, es la oportunidad que se presenta de abrir la transformación a distintos actores, pues otorga la posibilidad de introducir elementos que no fueron necesariamente expuestos en el último desastre. Esto, es en nuestra opinión una de las llaves para obtener un proceso de reconstrucción que mire hacia adelante, incorporando otras soluciones y observando de forma integral la configuración de la vulnerabilidad. Es decir, esto permitiría incluir en el proceso de metamorfosis, aquellos factores de riesgo que de alguna manera pasan bajo el radar cuando ocurre un desastre.

Estimamos necesario además, presentar soluciones al sistema de PPD actual en Chile a la luz de las mejores prácticas internacionales y políticas globales (Banco Interamericano de Desarollo, 2015; IFRC, 2015; UNASUR, 2015; UNISDR, 2015) para reducir el riesgo de desastres, pues el actual proyecto de ley y la política para la GRD carecen de alguno de estos elementos, como financiamiento adecuado.

El actual proyecto no tiene aumentos significativos en el presupuesto del Servicio Nacional de Gestión de Riesgo y Emergencias, lo que incluso llevó al rechazo de proyecto, en la Comisión de Defensa del Senado (Senado de Chile, 2015a). Esto, junto con el hecho de que actual presupuesto de ONEMI es sólo el 0,03\% del presupuesto nacional, muy por debajo de otros países.

De todas formas, no sólo es primordial aumentar el presupuesto de las instituciones destinadas a coordinar e implementar las acciones para reducir el riesgo de desastres. Se requiere también establecer mecanismos de transferencia de riesgo, los cuales constituyen una estrategia hacia el desarrollo sostenible. Un ejemplo fue el terremoto del 2010, donde el extraordinario proceso (Useem, Kunreuther, \& Michel-Kerjan, 2015) de recuperación se debió en gran parte a la penetración del mercado de seguros. No obstante, también es cierto que el Estado de Chile tuvo que recurrir a reformas tributarias y ajustes en el presupuesto nacional para financiar la reconstrucción, por lo que urge explorar instrumentos modernos de financiamiento de riesgo como los bonos de catástrofe. Por ende, la real transformación del sistema debe ser apoyada con financiamiento adecuado para cambiar el foco desde la gestión de desastre a la gestión prospectiva del riesgo (Lavell, Mansilla, \& Smith, 2003) y el desarrollo sostenible.

La profundidad del proceso de cambio depende en gran parte de una reconstrucción que mejore las condiciones previas, reconstruir mejor (Kennedy, Ashmore, Babister, \& Kelman, 2008; UNISDR, 2015). Mas, no basta con mejorar las condiciones previas, sino que se debe proyectar hacia adelante (Mena, 2016) el proceso de recuperación. Esto requiere incluir en el actual proyecto de ley todas las fases del ciclo de la GRD; incluyendo la reconstrucción y asegurando la prevención como el proyecto hoy contempla, gracias al proceso legislativo (Senado de Chile, 2015b). 
Finalmente, una cultura preventiva también debe ser inclusiva. En este sentido, la investigación descubrió de forma no intencionada, brechas de género relevantes. La selección de profesionales para entrevistar reveló una disparidad sustancial de género en los tomadores de decisión e individuos que trabajan en GRD. Por ejemplo, cuando ocurrió el Terremoto del 2010, ONEMI era dirigida por Carmen Fernández. Hoy, no hay mujeres en cargos directivos nacionales en esta organización y de los 15 directores regionales, sólo 3 son mujeres. Así también, el proyecto de ley no tiene referencias explícitas a temas de género. Este descubrimiento nuevamente demuestra que el cambio de PPD para que sea con transformación, debe también atacar transversalmente las vulnerabilidades sociales, asegurando un acceso equitativo al diseño, implementación, gestión, y evaluación de este tipo de políticas públicas.

Entonces ¿Requería cambios el sistema de protección civil chileno luego del 27F? Sin dudas. ¿Se abrió una ventana de oportunidad para introducir los cambios? Esta investigación así lo reconoce. Sin embargo, la profundidad y extensión de las modificaciones es limitada. En Chile se observa un proceso de cambio sin transformación, caracterizado por Lavell (1996, p. 70) como " una creciente sofisticación y un proceso tecnológico limitado, esencialmente, a las fases de preparación y respuesta a las emergencias y desastres; lo ha sido también en sus alcances, precisamente por la injerencia de una estructura y funcionalidad macro estatal, de características altamente centralizadas, que ha cohibido una eficaz descentralización participativa en el desarrollo de los componentes de la gestión de los desastres". Por ende, si bien se reconoce la apertura de una ventana de oportunidad, los cambios que ésta produjo no han dado pie a una metamorfosis del sistema. No existe una transformación en la lógica de las acciones, el sistema chileno aún adolece gestión prospectiva del riesgo.

La explicación más plausible al fenómeno anterior es la carencia de emprendedores de política pública de desastre, que pueden acoplar las tres corrientes: políticas -soluciones-, problemas y política, para crear un paquete que permita introducir cambio con transformación en las políticas públicas de desastre. El resultado, un sistema donde el foco en la respuesta aún prevalece sobre la prevención, el sesgo sísmico restringe una aproximación multi-amenaza y, el énfasis aún está en la amenaza, no en las vulnerabilidades de la sociedad chilena, haciendo por el momento difícil una metamorfosis hacia la reducción del riesgo y el desarrollo sostenible.

\section{REFERENCIAS}

Alexander, D. (2002). Principles of Emergency Planning and Management. Edinburgh: Terra Publishing.

Banco Interamericano de Desarollo. (2015). Índice de Gobernabilidad y Políticas Públicas en Gestión de Riesgo de Desastres (iGOPP): informe nacional Chile. Washington D.C. Obtenido de https://publications.iadb.org/bitstream/handle/11319/7239/iGOPP_Argentina.PDF?sequence=1

Beck, U. (2015). Emancipatory catastrophism: What does it mean to climate change and risk society? Current Sociology, 63(1), 75-88.

BID. (2015). Indicadores de Riesgo de Desastre y Gestión de Riesgos Programa para América Latina y el Caribe, Chile. New York. Obtenido de http://idbdocs.iadb.org/wsdocs/getdocument. aspx?docnum $=35160024$

Birkland, T. (1997). After disaster: agenda setting, public policy, and focusing events. Washington D.C.: Georgetown University Press.

Birkland, T. (2006). Lessons of Disaster: Policy Change after Catastrophic Events. Washington: Georgetown University Press.

Birkmann, J., Buckle, P., Jaeger, J., Pelling, M., Setiadi, N., Garschagen, M., ... Kropp, J. (2010). Extreme events and disasters: A window of opportunity for change? Analysis of organizational, institutional and political changes, formal and informal responses after mega-disasters. Natural Hazards, 55(3), 637-655.

Cámara de Diputados de la República de Chile. (2012). Informe de la Comisión de Seguridad Ciudadana y de Drogas, constituida en investigadora de los hechos sucedidos en las horas posteriores al terremoto y maremoto del 27 de febrero de 2010. Valparaíso.

Davis, I., \& Alexander, D. (2016). Recovery from Disaster. Abingdon: Routledge. 
DIPECHO. (2012). Análisis de riesgos de desastres en Chile. Santiago de Chile.

Gobierno de Chile. (2011). Proyecto de ley que establece el Sistema Nacional de Emergencia y Protección Civil y crea la Agencia Nacional de Protección Civil.

Gobierno de Chile. (2013). Informe Nacional del Progreso en la Implementación del Marco de Acción de Hyogo (2011-2013).

IFRC. (2015). The Checklist on Law and Disaster Risk Reduction. Geneva. Obtenido de http://www.ifrc. org/PageFiles/115542/The-checklist-on-law-and-drr.pdf

Jones, M. D., Peterson, H. L., Pierce, J. J., Herweg, N., Bernal, A., Lamberta Raney, H., \& Zahariadis, N. (2016). A River Runs Through It: A Multiple Streams Meta-Review. Policy Studies Journal, 44(1), $13-36$.

Kappes, M. S., Keiler, M., von Elverfeldt, K., \& Glade, T. (2012). Challenges of analyzing multi-hazard risk: A review. Natural Hazards, 64(2), 1925-1958. https://doi.org/10.1007/s11069-012-0294-2

Kennedy, J., Ashmore, J., Babister, E., \& Kelman, I. (2008). The meaning of “build back better”: Evidence From post-tsunami Aceh and Sri Lanka. Journal of Contingencies and Crisis Management, 16(1), 24-36.

Kilpatrick, D. G. (200o). Definitions of Public Policy and the Law. Obtenido el 17 de Febrero, 2016, de https://mainweb-v.musc.edu/vawprevention/policy/definition.shtml

Kingdon, J. (1995). Agendas, Alternatives, and Public Policies (2nd ed.). Boston: Harper Collins College Publishers.

Lavell, A. (1996). Costa Rica: cambio sin transformación los límites de un paradigma. En A. Lavell \& E. Franco (Eds.), Estado, sociedad y gestión de los desastres en América Latina. En busca del paradigma perdido (p. 405). La Red.

Lavell, A., Mansilla, E., \& Smith, D. (2003). La Gestión Local del Riesgo. Nociones y Precisiones en torno al Concepto y la Práctica. CEPREDENAC-PNUD. Panamá: PNUD. Obtenido de http://www.desenredando.org/public/libros/20o6/ges_loc_riesg/gestion_riesgo_espanol.pdf

Manyena, S. B. (2013). Disaster event: Window of opportunity to implement global disaster policies? Jàmbá: Journal of Disaster Risk Studies, 5(1), 1-10.

McCombs, M. E., \& Shaw, D. L. (1972). The Agenda-Setting Function of Mass Media. The Public Opinion Quarterly, 36(2), 176-187.

Mena, C. (2014). Informe Proyecto de Ley Boletín 7550-o6: Establece el Sistema Nacional de Emergencia y Protección Civil y crea la Agencia Nacional de Protección Civil. Santiago.

Mena, C. Building Back or Forward Better? Disaster Public Policy in Chile after the 27 February 2010 Earthquake. (2016). London: Unpublished manuscript.

OECD. (2012). Disaster Risk Assessment and Risk Financing. A G2o/OECD methodological framework. New York.

Olson, R. S., Sarmiento Prieto, J. P., \& Hoberman, G. (2010). Disaster Risk Reduction, Public Accountability, and the Role of the Media: Concepts, Cases, and Conclusions. Background Paper Prepared for the 2011 Global Assessment Report on Disaster Risk Reduction.

ONEMI. (2014). Política Nacional para la Gestión de Riesgo de Desastres. Santiago. Obtenido de http:// siac.onemi.gov.cl/documentos/POLITICA_NACIONAL_PARA_LA_RRD.pdf

Overseas Development Institute, \& The World Bank. (2015). Unlocking the "Triple Dividend" of Resilience. London.

Pelling, M., \& Dill, K. (2006). "Natural” Disasters as Catalysts of Political Action. Media Development, 53(4), 7. Obtenido de http://burmalibrary.org/docs4/Pelling-Disasters-1.pdf

Phillips, B. (2002). Qualitative Methods and Disaster Research. En R. A. Stallings (Ed.), Methods of Disaster Research (pp. 194-213). Bloomington: Xlibris Corporation.

Pidgeon, N., \& O’Leary, M. (2000). Man-made disasters: Why technology and organizations (sometimes) fail. Safety Science, 34(1-3), 15-30.

Platt, R. H. (1999). Disasters and democracy: the politics of extreme natural events. Washington D.C.: Island Press.

PreventionWeb. (2016). Chile - Disaster E Risk Profile. Obtenido el 17 de Febrero, 2016, de http://www. preventionweb.net/countries/chl/data/ 
Quarantelli, E. L. (1998). What is a disaster? Perspectives on the question. London: Routledge.

República de Chile. (1980). Constitución Política de la República.

República de Chile. (200o). Ley Orgánica Constitucional de Bases Generales de la Administración del Estado.

República de Chile. (2002). Decreto 156. Aprueba Plan Nacional de Protección Civil y deroga Decreto Supremo de Interior $N^{\circ} 155$, de 1977, que aprobó el Plan Nacional de Emergencia.

Revista Capital. (2016). Fondos de emergencia de Onemi crecieron siete veces desde el $27 F$.

Sabatier, P. (Ed.). (2007). Theories of the Policy Process (2nd ed.). Boulder: Westview Press.

Sabatier, P. A., \& Jenkins-Smith, H. C. (1993). Policy change and learning: an advocacy coalition approach. Boulder: Westview Press.

Sanhueza, C., Contreras, D., \& Denis, Á. (2012). Terremoto y sus efectos sobre el bienestar: un análisis multidimensional. Persona Y Sociedad, 26(1), 43-66.

Senado de Chile. (2015a). Comisión de Defensa rechaza idea de legislar sobre proyecto que crea sistema nacional de emergencia. Obtenido el 14 de Marzo, 2016, de http://www.senado.cl/comision-de-defensa-rechaza-idea-de-legislar-sobre-proyecto-que-crea-sistema-nacional-de-emergencia/prontus_senado/2015-11-10/235809.html

Senado de Chile. (2015b). Informe de la Comisión de Gobierno, Descentralización y Regionalización, recaído en el proyecto de ley, en segundo trámite constitucional, que establece el Sistema Nacional de Emergencia y Protección Civil y crea la Agencia Nacional de Protección Civil. Valparaíso.

Stallings, R. A. (1991). Feedback from the field. Disasters as Social Problems?: A dissenting view. International Journal of Mass Emergencies and Disasters, 9(1), 69-74.

Tierney, K., \& Oliver-Smith, A. (2012). Social Dimensions of Disaster Recovery. International Journal of Mass Emergencies and Disasters, 30(2), 123-146.

Tironi, M. (2014). Atmospheres of indagation: Disasters and the politics of excessiveness. Sociological Review, 62(S1), 114-134.

True, J. L., Jones, B. D., \& Baumgartner, F. R. (2007). Punctuated-Equilibrum Theory: Explaining Stability and Change in Public Policymaking. En P. A. Sabatier (Ed.), Theories of the Policy Process (2nd ed., pp. 155-187). Boulder: Westview Press.

UNASUR. (2015). Consideraciones para Fortalecer una Estrategia Suramericana para la Reducción del Riesgo de Desastres.

UNISDR. (2009). Terminología sobre Reducción del Riesgo de Desastres. Nueva York: Estrategia Internacional para la Reducción de Desastres de las Naciones Unidas. Obtenido de http://www. unisdr.org/files/7817_UNISDRTerminologySpanish.pdf

UNISDR. (2015). Sendai Framework for Disaster Risk Reduction 2015-2030. Geneva: UNISDR. Obtenido de http://www.unisdr.org/we/coordinate/sendai-framework

Useem, M., Kunreuther, H., \& Michel-Kerjan, E. (2015). Leadership dispatches: Chile’s extraordinary comeback from disaster. Stanford: Stanford University Press.

Wachinger, G., Renn, O., Begg, C., \& Kuhlicke, C. (2013). The risk perception paradox-implications for governance and communication of natural hazards. Risk Analysis, 33(6), 1049-1065.

Wisner, B. (2003). Changes in capitalism and global shifts in the distribution of hazard and vulnerability. En M. Pelling (Ed.), Natural Disasters and Development in a Globalizing World (p. 250). London: Routledge.

Wisner, B., Blaikie, P., Cannon, T., \& Davis, I. (2004). At Risk (2nd ed.). Abingdon: Routledge.

Wu, J. Y., \& Lindell, M. K. (2004). Housing reconstruction after two major earthquakes: the 1994 Northridge earthquake in the United States and the 1999 Chi-Chi earthquake in Taiwan. Disasters, 28(1), 63-81.

Zahariadis, N. (2007). The Multiple Streams Framework. Structure, Limitations, Prospects. En P. A. Sabatier (Ed.), Theories of the Policy Process (2nd ed., pp. 65-92). Boulder: Westview Press. 